\title{
AREA-WIDE DISTRIBUTION OF LEAD, COPPER, AND CADMIUM IN AIR PARTICULATES FROM CHICAGO AND NORTHWEST INDIANA*
}

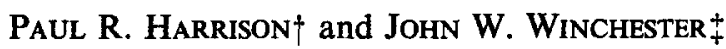 \\ Department of Meteorology and Oceanography, \\ University of Michigan, \\ Ann Arbor, Michigan 48104, U.S.A.
}

(First received 15 April 1971 and in final form 3 May 1971)

\begin{abstract}
Published air pollution emissions inventories for the urbanized and industrialized area along the southwestern shore of Lake Michigan include few chemical analyses of particulates, and estimates of the elemental composition of airborne solids may be made only indirectly and compared with NASN analyses from a few locations. As a first attempt to examine the area-wide distribution of specific chemical elements in this region, lead, copper, cadmium, and bismuth were determined in 24-h average samples collected on glass fiber filters at 50 stations throughout the region. Samples from most of the 50 stations were obtained from local air pollution control organizations for 6 different days from May to August 1968 and were analyzed electrochemically by highly sensitive anodic stripping voltammetry (ASV). Throughout the area the small variation of lead, generally a few micrograms per cubic meter of air, did not exceed that expected from the distribution of automobiles, the major source. Cadmium was generally 200 times lower without marked local variations and was close to the expected concentration if coal combustion is the major source. Copper was generally 20 times lower than lead throughout Chicago as expected if coal combustion is the major source of copper. However, certain stations in the northwest Indiana area showed reproducible anomalies where copper was 100 times greater than in Chicago and several times greater than lead at the same stations. The source of this anomalous copper has not been determined.
\end{abstract}

\section{INTRODUCTION}

UNTIL recently area-wide air pollution surveys of suspended particulate have commonly been made without determination of elcmental composition, primarily because of increased cost of chemical analysis over that of simply measuring weight of total particulate collected on a filter. However, as the present work has found, geographic variability in the atmospheric concentrations of certain elements composing the particulate may exceed that of total suspended particulate in an urban or industrial area, and detailed chemical data may be necessary to evaluate public health hazards or to locate sources and estimate their source strengths. We have carried out chemical analyses by anodic stripping voltammetry (ASV) of particulate sampled on glass fiber filters by several local control agencies in the Chicago-Northwest Indiana region for six 24-h periods during 1968: 21-22 May, 6 and 20 June, 9 July, 8 and 29 August. Data for $\mathrm{Pb}, \mathrm{Cd}$, and $\mathrm{Cu}$ are presented as well as data for $\mathrm{Bi}$ on the first day (21 May for Chicago and 22 May for Indiana).

One of the most extensive area-wide surveys was carried out in Nashville, Tennessee, (ZEIDBERG et al., 1961) utilizing 119 sampling stations distributed in and around the

* Presented at the Annual Meeting of the Air Pollution Control Association, St. Louis, Missouri, 15-19 June, 1970.

$\dagger$ Present address: Dept. of Environmental Control, 320 N. Clark St., Chicago, Illinois 60610, U.S.A.

$\ddagger$ Present address: Dept. of Oceanography, Florida State University, Tallahassee, Florida 32306, U.S.A. 
city. The parameters measured included spot tapes, total particulate, and sulfur dioxide, but no trace element analyses were reported. One of the first trace metal studies was by TABOR and WARNER (1958) where 17 metals in 28 cities were determined, although none of the sampling sites were adjacent to each other and few were simultaneous. More recently LeE and Jervis (1968) and BRAR et al, (1970) have determined by neutron activation analysis several trace metals in atmospheric particulate from Toronto, Canada, and Chicago, Illinois, respectively. The latter study reported concentrations of 21 metals in 22 samples taken simultaneously over a $24-\mathrm{h}$ period in April, 1968. Significant variations in concentrations were found, presumably reflecting variability in the distribution of industrial sources over the city. No data for Northwest Indiana were reported, and the relevance of meteorological conditions to the concentration patterns could not be determined from this one-day study. KNEIP et al. (1970) have just published a trace metal study of New York with results consistent with ours in the present investigation.

\section{EXPERIMENTAL}

In the present work we present data for $\mathrm{Pb}, \mathrm{Cd}, \mathrm{Cu}$, and $\mathrm{Bi}$ in aerosol particles from the Chicago and Northwest Indiana regions. This was a cooperative cffort mainly with the City of Chicago and the Northwest Indiana Air Resource Management

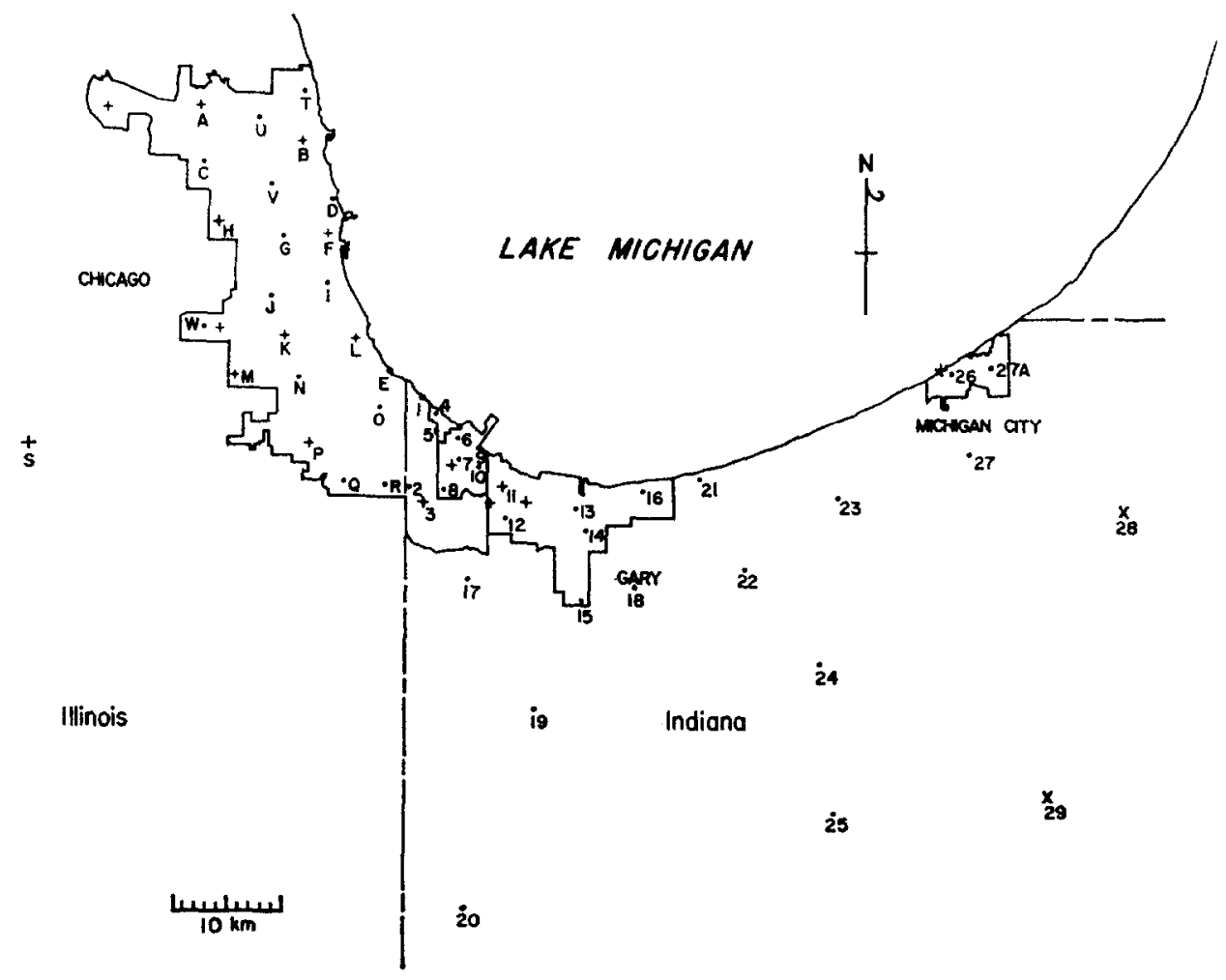

FIG. 1. Air sampling locations. 
Group, consisting of Hammond, East Chicago, Whiting, Gary, Michigan City, and Lake County, with Porter County and Valparaiso University also participating. From these groups we obtained data for $\mathrm{SO}_{2}$ and suspended particulate which we quote here as well as the samples which we analyzed in our laboratory for trace metals. FIGURE 1 shows the station designations in the area under study.

The six sampling days selected, all Tuesdays or Thursdays, give the greatest degree of simultaneity possible over the several jurisdictions involved. All samples were collected on standard $20 \times 25 \mathrm{~cm}(8 \times 10$ in.) glass fiber filter paper, used routinely for suspended particulate analysis. After each filter paper was weighed it was folded and placed in envelopes by the local agencies and stored in a filing cabinet until taken to our laboratory at the University of Michigan.

Our procedure consisted of the following steps: A $13 \mathrm{~cm}^{2}\left(2 \mathrm{in}^{2}\right)$ sample was cut as 2 equal-sized squares from the folded filter paper, and then slightly shredded and inserted into a standard $25 \mathrm{ml}$ narrow neck volumetric flask. The organics were dissolved by digesting in $4 \mathrm{ml}$ of perchloric acid and heated to $300^{\circ} \mathrm{C}$ for approximately $1 / 2 \mathrm{~h}$. The samples were then diluted to $25 \mathrm{ml}$ with water and allowed to sit for 1-2 days before analysis. Equivalent portions of blank filters obtained with the samples were treated in the same manner, although we found no evidence of significant contamination by the glass fiber for the elements measured.

The ASV technique, an electrochemical method akin to polarography, consists of electrodeposition of the trace elements from solution onto an electrode and then stripping by reversing the potential in a gradual sweep. The current caused by each element in turn re-entering the solution is recorded by a moving chart recorder, and the total charge collected, a measure of the element, is calculated by measurement of height or area of the current-voltage peaks characteristic for each element. MATSON (1968) has given a description of the electrode used consisting of a thin $\mathrm{Hg}$ film coating a paraffin-impregnated graphite rod, and the construction of the electrochemical cell and associated electronics. In general, the technique is sensitive to nanogram amounts of metals which form amalgams and is well suited to air pollution investigations.

An aliquot of each $25-\mathrm{ml}$ sample solution, representing about $0.3 \mathrm{~m}^{3}$ of air, was added to a vial of $10 \mathrm{ml}$ pre-treated solution and subjected to ASV analysis. In order to facilitate mass production of analyses a routine for handling samples, blanks, and standards in a cumulative fashion was devised and is described in detail by HARRISON (1970). To alleviate carryover from one sample to another each electrode system and vial were washed thoroughly and then an aliquot of blank plus a standard spike was added and run. The subsequent stripping was the sample itself with the former response subtracted from the sample run, thus eliminating contamination in the vial and by carryover from the previous analysis. Analytical control was achieved by running different cells at different times for the same filter paper and by using aliquots from various regions in the filter paper in replicate determinations. Due to the response characteristics of the apparatus the errors are variable according to the response to blank ratio. Since the ASV technique is being given its first large scale test in an air pollution survey application by this work, we regard the relative concentration variations over the network to be of greater significance, and absolute concentrations for individual samples may be in error by as much as a factor of 2 . 
TABle 1. AtMospheric Concentrations of LEAD $\left(\mathrm{ng} \mathrm{m}^{-3}\right)$

\begin{tabular}{|c|c|c|c|c|c|c|}
\hline Station & 21/22 May & 6 June & 20 June & 9 July & 8 Aug. & 29 Aug. \\
\hline 1 & 2000 & 800 & 1000 & 100 & 1000 & 1000 \\
\hline 2 & - & 2000 & 3000 & 2000 & 3000 & 2000 \\
\hline 3 & 3000 & 1000 & 3000 & 2000 & 2000 & 2000 \\
\hline 4 & 3000 & 1000 & 2000 & 800 & 2000 & 2000 \\
\hline 5 & 1000 & 900 & 2000 & 400 & 1000 & 1000 \\
\hline 6 & 2000 & 900 & 4000 & 600 & 4000 & 2000 \\
\hline 7 & - & - & 3000 & 700 & 3000 & 2000 \\
\hline 8 & 1000 & 700 & 2000 & 1000 & 1000 & 1000 \\
\hline 9 & 2000 & 500 & 600 & 2000 & 2000 & 500 \\
\hline 10 & 2000 & 300 & 800 & 1000 & 700 & 600 \\
\hline 11 & 2000 & 1000 & 2000 & - & - & - \\
\hline 12 & 4000 & 700 & 2000 & - & - & - \\
\hline 13 & 6000 & 2000 & 3000 & - & - & - \\
\hline 14 & - & - & - & $\ldots$ & - & - \\
\hline 15 & 1000 & 500 & 2000 & - & - & - \\
\hline 16 & 3000 & 800 & $\ldots$ & - & $\ldots$ & - \\
\hline 17 & 1000 & 500 & 2000 & 900 & 2000 & 700 \\
\hline 18 & 400 & 300 & 800 & 500 & 100 & 2000 \\
\hline 19 & 300 & 400 & 400 & - & 100 & 3000 \\
\hline 20 & 900 & 700 & 1000 & 1000 & 600 & 1000 \\
\hline 21 & - & - & 1000 & 500 & 600 & - \\
\hline 22 & - & - & 1500 & 400 & 1000 & - \\
\hline 23 & - & - & 700 & 100 & 500 & 700 \\
\hline 24 & - & - & 1500 & 500 & 300 & 500 \\
\hline 25 & - & - & 500 & 300 & 200 & - \\
\hline 26 & 1000 & - & 1000 & 700 & 800 & 500 \\
\hline 27 & - & - & - & 300 & 400 & 800 \\
\hline A & 7000 & 1000 & 5000 & 2000 & 3000 & 4000 \\
\hline B & 4000 & 2000 & 2000 & 800 & 4000 & 4000 \\
\hline C & 5000 & 300 & 2000 & 600 & 2000 & 3000 \\
\hline D & 3000 & 2000 & 300 & 2000 & 4000 & 3000 \\
\hline $\mathrm{E}$ & 4000 & - & - & - & 1500 & 5000 \\
\hline $\mathrm{F}$ & 6000 & 2000 & 3000 & - & 3000 & 4000 \\
\hline G & 4000 & 2000 & 4000 & 1500 & - & 3000 \\
\hline H & 2000 & 1000 & 3000 & 700 & 3000 & 4000 \\
\hline I & - & - & - & - & - & 2000 \\
\hline $\mathrm{J}$ & 6000 & 900 & 2000 & - & 2000 & - \\
\hline $\mathrm{K}$ & 5000 & 1000 & 2000 & 500 & 2000 & 2000 \\
\hline $\mathrm{L}$ & 3000 & 1500 & 2000 & 200 & 4000 & 2000 \\
\hline$\vec{M}$ & 6000 & 1000 & 2000 & 400 & 2000 & 4000 \\
\hline $\mathrm{N}$ & 5000 & 1000 & 3000 & 600 & 2000 & 2000 \\
\hline$\Theta$ & 6000 & 1000 & 2000 & 500 & 2000 & 1000 \\
\hline $\mathbf{P}$ & 5000 & 1000 & 4000 & 600 & 2000 & 3000 \\
\hline$Q$ & 4000 & 700 & 2000 & 700 & - & 2000 \\
\hline $\mathrm{R}$ & 4000 & - & 2000 & 300 & 2000 & - \\
\hline$T$ & 3000 & 1000 & 2000 & 800 & - & - \\
\hline $\mathrm{U}$ & 4000 & 1500 & 3000 & 1000 & 3000 & - \\
\hline V & 4000 & 1000 & 4000 & 1000 & 3000 & 2000 \\
\hline w & 4000 & 800 & 2000 & - & 2000 & 3000 \\
\hline Ship & - & - & $\ldots$ & 700 & - & - \\
\hline
\end{tabular}

Individual data points are reliable to a factor of 2 . 
TABLE 2. ATMOSPHERIC CONCENTRATIONS OF CADMIUM (ng $\mathrm{m}^{-3}$ )

\begin{tabular}{|c|c|c|c|c|c|c|}
\hline Station & 21/22 May & 6 June & 20 June & 9 July & 8 Aug. & 29 Aug. \\
\hline 1 & 50 & 5 & 30 & 10 & ** & 10 \\
\hline 2 & - & $* *$ & 20 & 20 & 10 & 20 \\
\hline 3 & 10 & $* *$ & $* *$ & 50 & ** & 30 \\
\hline 4 & 30 & ** & $* *$ & 5 & 10 & 8 \\
\hline 5 & 40 & 10 & 10 & 50 & $* *$ & 7 \\
\hline 6 & 10 & 8 & 20 & 10 & 10 & 10 \\
\hline 7 & - & - & 8 & 60 & 10 & 10 \\
\hline 8 & 15 & $* *$ & 5 & 10 & 8 & $* *$ \\
\hline 9 & 30 & 7 & 10 & 20 & ** & $* *$ \\
\hline 10 & 20 & $* *$ & 3 & 6 & $* *$ & $* *$ \\
\hline 11 & 10 & 40 & 6 & - & - & - \\
\hline 12 & 70 & 10 & $* *$ & - & - & - \\
\hline 13 & 30 & 30 & $* *$ & - & - & - \\
\hline 14 & - & - & - & - & - & - \\
\hline 15 & 15 & $* *$ & $* *$ & - & - & - \\
\hline 16 & 7 & 9 & - & - & - & - \\
\hline 17 & 10 & $* *$ & ** & $* *$ & $* *$ & $* *$ \\
\hline 18 & $* *$ & 20 & $* *$ & 10 & $* *$ & 20 \\
\hline 19 & 9 & $* *$ & $* *$ & - & $* *$ & 40 \\
\hline 20 & 10 & 6 & 80 & 20 & 5 & 20 \\
\hline 21 & $\cdots$ & - & 30 & 40 & $* *$ & - \\
\hline 22 & - & - & 30 & 10 & $* *$ & - \\
\hline 23 & - & - & 30 & 30 & $* *$ & $* *$ \\
\hline 24 & - & - & 20 & 7 & 20 & 10 \\
\hline 25 & - & - & 30 & $* *$ & ** & - \\
\hline 26 & 5 & - & *** & 7 & 10 & $* *$ \\
\hline 27 & - & - & - & $* *$ & 10 & 20 \\
\hline A & $* *$ & $* *$ & 20 & 20 & 10 & 30 \\
\hline B & 30 & 30 & $* *$ & 10 & 30 & 30 \\
\hline C & 30 & 6 & 10 & *** & 20 & $* *$ \\
\hline D & $\mathbf{M}$ & 20 & *** & 20 & ** & 20 \\
\hline E & 60 & - & - & - & 15 & 40 \\
\hline$\overline{\mathbf{F}}$ & 20 & 10 & 10 & - & 10 & 5 \\
\hline $\mathbf{G}$ & 50 & 10 & $* *$ & 10 & - & 15 \\
\hline $\mathbf{H}$ & ** & $* *$ & 20 & 8 & 30 & 40 \\
\hline I & - & - & - & - & - & $* *$ \\
\hline$J$ & 80 & $* *$ & 10 & - & 8 & - \\
\hline $\mathbf{K}$ & 20 & ** & 8 & 10 & 10 & 10 \\
\hline L & 30 & 15 & $* *$ & $* *$ & 20 & 30 \\
\hline $\mathbf{M}$ & 40 & 6 & 9 & $* *$ & 20 & $* *$ \\
\hline $\mathbf{N}$ & 10 & 8 & 20 & 9 & 10 & $* *$ \\
\hline$\Theta$ & 50 & 10 & 10 & *** & 10 & 7 \\
\hline $\mathbf{P}$ & 5 & 20 & 9 & 20 & $* *$ & 30 \\
\hline$Q$ & 30 & 10 & 6 & 15 & - & 6 \\
\hline $\mathbf{R}$ & 20 & - & 7 & ** & 10 & - \\
\hline $\mathbf{T}$ & 10 & 10 & 9 & ** & ** & - \\
\hline$\dot{U}$ & 9 & $* *$ & 10 & ** & 20 & - \\
\hline V & 40 & 7 & 40 & 6 & 30 & 30 \\
\hline W & 40 & 9 & 10 & - & $* *$ & $* *$ \\
\hline Ship & - & - & - & 20 & - & - \\
\hline
\end{tabular}

Individual data points are reliable to a factor of 2 . $* *=\leqslant 5$. 
TABLE 3. ATMOSPHERIC CONCENTRATIONS OF COPPER $\left(\mathrm{ng} \mathrm{m}^{-3}\right)$

\begin{tabular}{|c|c|c|c|c|c|c|}
\hline Station & 21/22 May & 6 June & 20 June & 9 July & 8 Aug. & 29 Aug. \\
\hline 1 & 70 & 300 & 400 & 150 & 150 & 300 \\
\hline 2 & - & 100 & 1000 & 150 & 200 & 150 \\
\hline 3 & 300 & 200 & 2000 & 200 & 80 & 600 \\
\hline 4 & 900 & 2000 & 200 & 80 & 600 & 400 \\
\hline 5 & 1000 & 3000 & 600 & 60 & 500 & 200 \\
\hline 6 & 2000 & 7000 & $(7000)$ & 2000 & 2000 & 4000 \\
\hline 7 & - & - & 1000 & 300 & 1000 & 600 \\
\hline 8 & 5000 & 9000 & 5000 & 2000 & 3000 & 9000 \\
\hline 9 & 7000 & 5000 & 9000 & 7000 & 10000 & 10000 \\
\hline 10 & 4000 & 5000 & 1500 & 2000 & $(9000)$ & 10000 \\
\hline 11 & 300 & 700 & 400 & - & - & - \\
\hline 12 & $* * *$ & 300 & 500 & -- & $\ldots$ & - \\
\hline 13 & 80 & 700 & 200 & - & - & - \\
\hline 14 & - & - & - & - & - & - \\
\hline 15 & 200 & 200 & 150 & - & - & - \\
\hline 16 & 100 & 200 & - & - & - & - \\
\hline 17 & 150 & 200 & 150 & 200 & 30 & 70 \\
\hline 18 & 300 & 200 & 300 & 200 & $* * *$ & 100 \\
\hline 19 & 400 & 700 & 400 & - & 70 & 100 \\
\hline 20 & 150 & 200 & 100 & 200 & 40 & 100 \\
\hline 21 & - & - & 100 & 70 & 70 & - \\
\hline 22 & - & - & 300 & 70 & 70 & -- \\
\hline 23 & - & - & 90 & $* * *$ & 100 & 100 \\
\hline 24 & - & - & 500 & 60 & 100 & 70 \\
\hline 25 & - & - & 200 & 60 & 90 & - \\
\hline 26 & 80 & $\ldots$ & 300 & 200 & 400 & 200 \\
\hline 27 & - & - & $* * *$ & 300 & 300 & 200 \\
\hline A & 70 & $* * *$ & 600 & 100 & 100 & 300 \\
\hline B & 300 & 200 & 70 & 200 & 300 & 150 \\
\hline $\mathrm{C}$ & 100 & 100 & 100 & 20 & $* *$ & 100 \\
\hline D & 150 & 70 & 30 & 100 & 200 & 200 \\
\hline E & 400 & - & - & - & 500 & 1000 \\
\hline $\bar{F}$ & 200 & $* * *$ & 80 & - & 100 & 100 \\
\hline $\mathrm{G}$ & 400 & 100 & 70 & 200 & $\ldots$ & 200 \\
\hline $\mathrm{H}$ & 500 & 20 & 80 & 20 & 100 & $* * *$ \\
\hline I & - & - & - & - & - & 200 \\
\hline $\mathbf{J}$ & 200 & 100 & $* * *$ & - & 200 & - \\
\hline $\mathrm{K}$ & 200 & $* * *$ & $* * *$ & 200 & 100 & 100 \\
\hline $\mathrm{L}$ & 200 & 100 & 70 & $* * *$ & 800 & 200 \\
\hline $\mathbf{M}$ & 80 & $* * *$ & 60 & $* * *$ & 600 & 90 \\
\hline $\mathbf{N}$ & 100 & 80 & 300 & 60 & 300 & 100 \\
\hline$\Theta$ & 300 & 200 & 200 & 100 & 200 & 200 \\
\hline $\mathrm{P}$ & 300 & 100 & 200 & 200 & 200 & 200 \\
\hline$Q$ & 900 & 30 & 100 & 200 & - & 200 \\
\hline $\mathrm{R}$ & 500 & $* * *$ & 200 & - & 200 & - \\
\hline $\mathrm{T}$ & 200 & 200 & 200 & 30 & $* * *$ & - \\
\hline U & 200 & 200 & 200 & 20 & 300 & - \\
\hline V & 600 & 100 & 400 & 80 & 200 & 200 \\
\hline W & 200 & 40 & 100 & $\ldots$ & 200 & 200 \\
\hline Ship & - & - & - & 100 & - & - \\
\hline
\end{tabular}

Individual data points are reliable to a factor of 2 .

$* * *=\leqslant 20$. 
Area-wide Distribution of Lead, Copper, and Cadmium in Air Particulates

TABle 4. AtMosPheric CONCENTRATIONS OF Bismuth $\left(\mathrm{ng} \mathrm{m}^{-3}\right)$

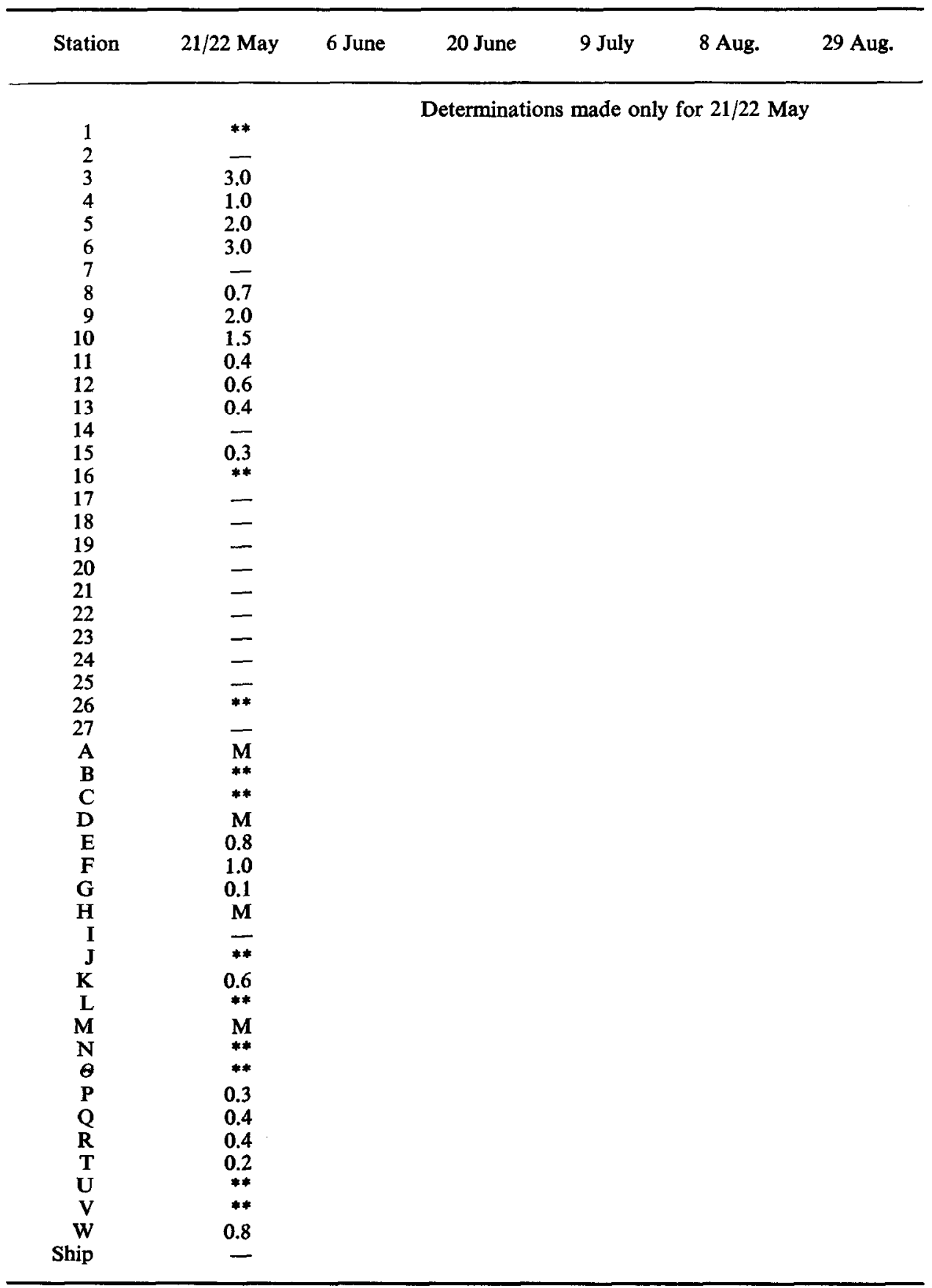

Individual data points are reliable to a factor of 2 .

$* *=\leqslant 0.05$. 
TABle 5. AtMospheric CONCENTRATIONS OF total SUSPENDED PARTiCulate $\left(\mu \mathrm{g} \mathrm{m}^{-3}\right)$

\begin{tabular}{|c|c|c|c|c|c|c|}
\hline Station & 21/22 May & 6 June & 20 June & 9 July & 8 Aug. & 29 Aug. \\
\hline 1 & 115 & 170 & 117 & 32 & 198 & 148 \\
\hline 2 & - & 218 & 344 & 64 & 161 & 92 \\
\hline 3 & 81 & 131 & 256 & 139 & 148 & 167 \\
\hline 4 & 126 & 143 & 128 & 54 & 152 & 145 \\
\hline 5 & 140 & 169 & 278 & 66 & 156 & 242 \\
\hline 6 & 142 & 201 & 382 & 125 & 256 & 233 \\
\hline 7 & - & - & 332 & 193 & 191 & 174 \\
\hline 8 & 62 & 108 & 210 & 105 & 91 & 122 \\
\hline 9 & 83 & 115 & 134 & 148 & 122 & 109 \\
\hline 10 & 78 & 109 & 198 & - & 100 & 96 \\
\hline 11 & 76 & 153 & 228 & - & - & - \\
\hline 12 & 128 & 179 & 249 & $\ldots$ & $\ldots$ & -- \\
\hline 13 & 120 & 187 & 255 & - & $\ldots$ & - \\
\hline 14 & - & $\ldots$ & - & _- & - & - \\
\hline 15 & 46 & 136 & 100 & - & - & - \\
\hline 16 & 80 & 154 & 85 & - & - & - \\
\hline 17 & 66 & 109 & 117 & 142 & - & - \\
\hline 18 & 98 & 86 & 107 & 64 & 17 & - \\
\hline 19 & 55 & 98 & 70 & - & 46 & - \\
\hline 20 & 78 & 96 & 88 & 126 & 62 & - \\
\hline 21 & - & - & 77 & 44 & 40 & 65 \\
\hline 22 & - & $\ldots$ & 104 & 55 & 49 & 65 \\
\hline 23 & - & - & 66 & 27 & 39 & 57 \\
\hline 24 & - & - & - & 32 & 38 & 81 \\
\hline 25 & - & - & 81 & - & 43 & 185 \\
\hline 26 & 68 & - & 64 & 63 & 79 & 45 \\
\hline 27 & - & - & - & 49 & 84 & 69 \\
\hline A & 88 & 174 & 141 & 71 & 138 & 107 \\
\hline B & 113 & 212 & 116 & 105 & 239 & 214 \\
\hline $\mathrm{C}$ & 114 & 55 & 150 & 49 & 132 & 139 \\
\hline D & 125 & 261 & 19 & 60 & 243 & 188 \\
\hline $\mathbf{E}$ & 144 & - & - & - & 305 & 952 \\
\hline $\bar{F}$ & 153 & 206 & 169 & - & 185 & 218 \\
\hline $\mathrm{G}$ & 134 & 181 & 172 & 152 & - & 143 \\
\hline $\mathrm{H}$ & 50 & 176 & 236 & 86 & 182 & 186 \\
\hline 1 & - & - & - & - & - & 181 \\
\hline $\mathrm{J}$ & 166 & 205 & 259 & - & 166 & - \\
\hline $\mathrm{K}$ & 154 & 139 & 245 & 103 & 158 & 134 \\
\hline L & 180 & 229 & 180 & 43 & 225 & 218 \\
\hline$M$ & 139 & 130 & 183 & 98 & 170 & 195 \\
\hline $\mathbf{N}$ & 142 & 115 & 211 & 86 & 154 & 136 \\
\hline$\Theta$ & 163 & 205 & 263 & 108 & 174 & 178 \\
\hline P & 141 & 153 & 286 & 51 & 177 & 214 \\
\hline$Q$ & 195 & 161 & 408 & 109 & - & 235 \\
\hline $\mathbf{R}$ & 143 & - & 241 & 110 & 130 & - \\
\hline $\mathrm{T}$ & 92 & 152 & 81 & 67 & 132 & - \\
\hline $\mathrm{U}$ & 75 & 172 & 121 & 74 & 146 & - \\
\hline V & 114 & 186 & 190 & 100 & 196 & 148 \\
\hline w & 131 & 155 & 216 & - & 161 & 185 \\
\hline
\end{tabular}

All data furnished by the local agencies. 
TABle 6. ATMOSPHERIC CONCENTRATIONS OF $\mathrm{SO}_{2}$ (ppb)

\begin{tabular}{|c|c|c|c|c|c|c|}
\hline Station & 21/22 May & 6 June & 20 June & 9 July & 8 Aug. & 20 Aug \\
\hline \multicolumn{7}{|l|}{1} \\
\hline $\begin{array}{l}3 \\
4\end{array}$ & 17 & 21 & 62 & 69 & 3 & - \\
\hline $\begin{array}{l}5 \\
6\end{array}$ & 13 & 24 & - & 48 & 4 & - \\
\hline 7 & - & - & 2 & - & 1 & - \\
\hline $\begin{array}{l}8 \\
9\end{array}$ & \multicolumn{5}{|c|}{9} & - \\
\hline \multicolumn{7}{|l|}{10} \\
\hline $\begin{array}{l}11 \\
12\end{array}$ & \multicolumn{4}{|c|}{12} & - & - \\
\hline \multicolumn{7}{|l|}{$\begin{array}{l}13 \\
15\end{array}$} \\
\hline \multicolumn{7}{|l|}{16} \\
\hline \multicolumn{7}{|l|}{17} \\
\hline \multicolumn{7}{|l|}{18} \\
\hline \multicolumn{7}{|l|}{20} \\
\hline 21 & - & 9 & 2 & 1 & 1 & 14 \\
\hline 22 & - & - & 1 & $* *$ & 3 & $* *$ \\
\hline 23 & - & 8 & 4 & 1 & 4 & 11 \\
\hline $\begin{array}{l}24 \\
25 \\
26 \\
27\end{array}$ & - & - & - & 1 & 2 & - \\
\hline $\mathbf{A}$ & 75 & 5 & 10 & 8 & 3 & 4 \\
\hline B & 14 & 1 & 27 & 35 & 3 & 26 \\
\hline$\overline{\mathrm{C}}$ & 54 & 17 & 10 & 1 & 4 & 6 \\
\hline $\mathrm{D}$ & 22 & 67 & 12 & 30 & 13 & 11 \\
\hline \multicolumn{7}{|l|}{ E } \\
\hline$F$ & 10 & 39 & 9 & 13 & 9 & 34 \\
\hline $\mathbf{G}$ & 32 & 45 & $* *$ & 6 & 3 & 16 \\
\hline \multirow{2}{*}{\multicolumn{7}{|c|}{$\underset{\mathbf{I}}{\mathbf{H}}$}} \\
\hline & & & & & & \\
\hline$\hat{K}$ & 80 & 12 & 14 & 12 & $\begin{array}{r}0 \\
24\end{array}$ & 1 \\
\hline I & 23 & 15 & 29 & 1 & 27 & - \\
\hline $\mathbf{M}$ & 1 & - & 6 & 1 & 15 & 32 \\
\hline $\mathbf{N}$ & 1 & - & 25 & 12 & 4 & 39 \\
\hline$\theta$ & 1 & - & 20 & 4 & 3 & 20 \\
\hline $\mathbf{P}$ & 1 & - & $* *$ & 1 & 2 & 5 \\
\hline$Q$ & 24 & 8 & 51 & 7 & 2 & 44 \\
\hline $\mathbf{R}$ & 17 & 7 & 51 & 4 & 2 & - \\
\hline $\mathbf{T}$ & 1 & 2 & 12 & 12 & 28 & 16 \\
\hline \multicolumn{7}{|l|}{$\mathbf{U}$} \\
\hline $\mathbf{V}$ & & & & & & \\
\hline $\mathbf{W}$ & 4 & 9 & 13 & 24 & 22 & 20 \\
\hline
\end{tabular}

$* *=\leqslant 0.5$.

All data furnished by the local agencies. 


\section{RESULTS}

TABLES 1-6 represent the results for the six days of lead, cadmium, copper, and bismuth by our work and of sulfur dioxide and suspended particulate data supplied to us by the local control agencies.

Concentrations are given as $\mathrm{ng} \mathrm{m^{-3 }}$ of air sampled, the lines ( ) signify no sample was taken, the asterisks (*) signify weak signals near blank level, and $\mathbf{M}$ indicates data missing for various reasons.

All data have been plotted on maps and are given by HaRRISON (1970). Since a great deal of similarity is found among the 6 days of the study, in spite of differences in winds, we have selected only one of the days for plotting here. Thursday, 6 June is the day with the least variable wind direction, and FIG. 2 shows the wind roses for four

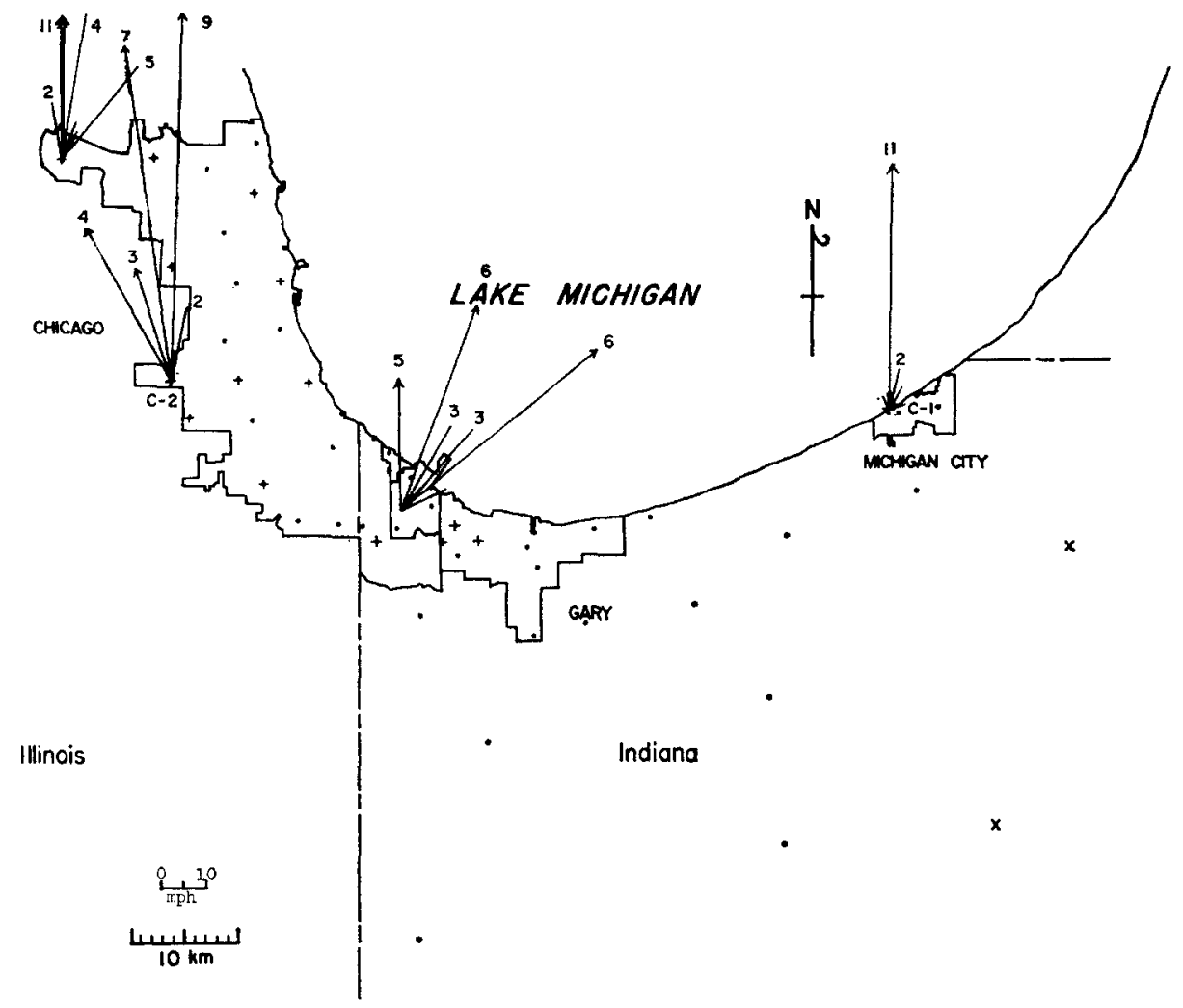

Fig. 2. Wind, Thursday, 6 June, 1968.

meteorological stations as lines in the direction toward which the wind was blowing for the number of hours indicated with lengths proportional to wind speed. C (calm) implies $<1.5 \mathrm{~m} \mathrm{~s}^{-1}$ winds. Lead in Fig. 3 shows a mean concentration of $1-2 \mu \mathrm{g} \mathrm{m}^{-3}$ and follows the average traffic density with no obvious strong local sources. Cadmium in Fig. 4 has a mean value near $10 \mathrm{ng} \mathrm{m}^{-3}$ and does not vary markedly with location although the variability is somewhat greater than for lead. Copper, in FIG. 5 shows a mean value near $100 \mathrm{ng} \mathrm{m}^{-3}$ over Chicago but has an anomalous "hot spot" in East 


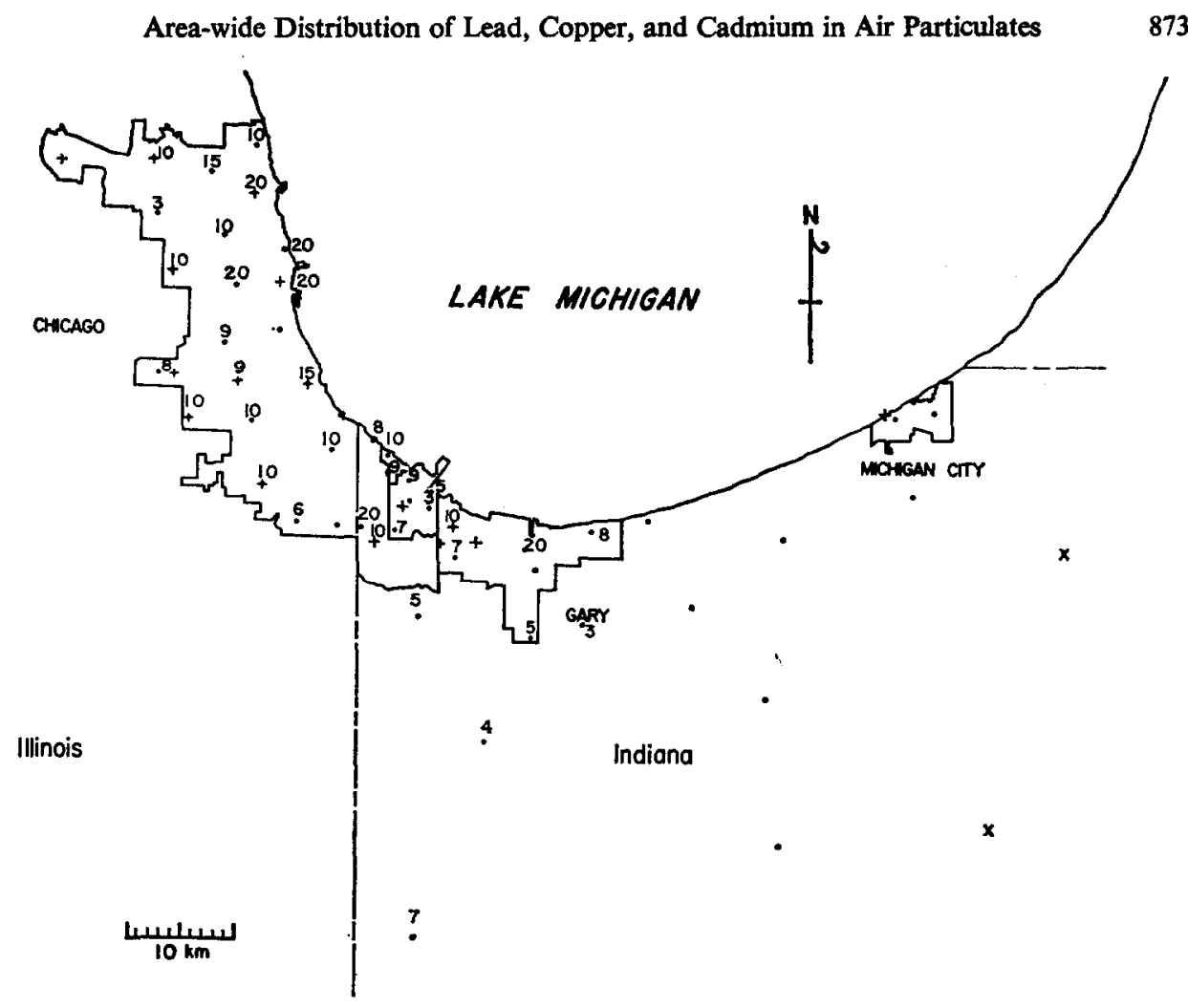

FIG. 3. Concentration of lead ( $\left.\mu \mathrm{g} \mathrm{m}^{-3} \times 10\right)$, Thursday, 6 June, 1968.

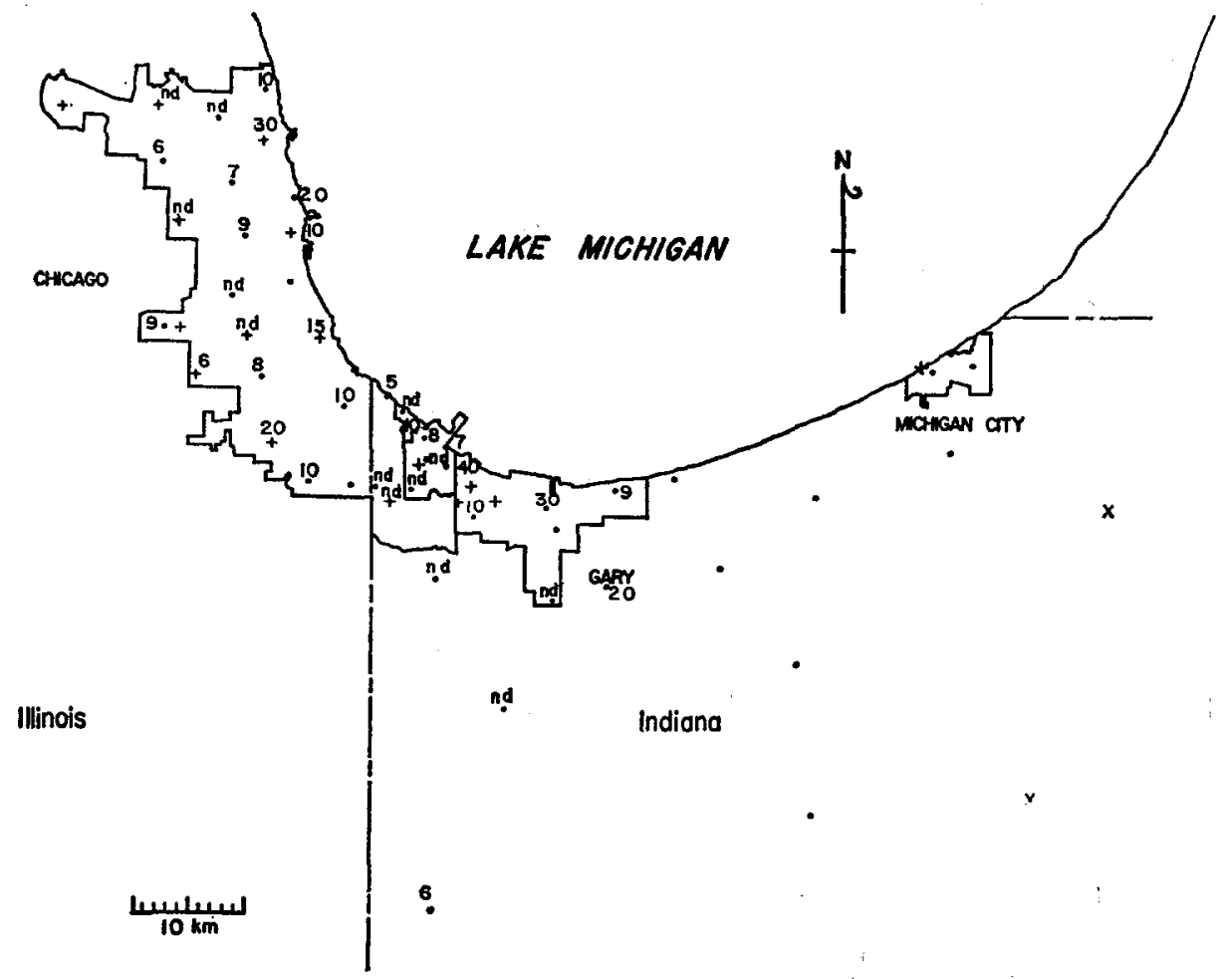

FIG. 4. Concentration of cadmium (ng m ${ }^{-3}$ ), Thursday, 6 June, 1968 . 


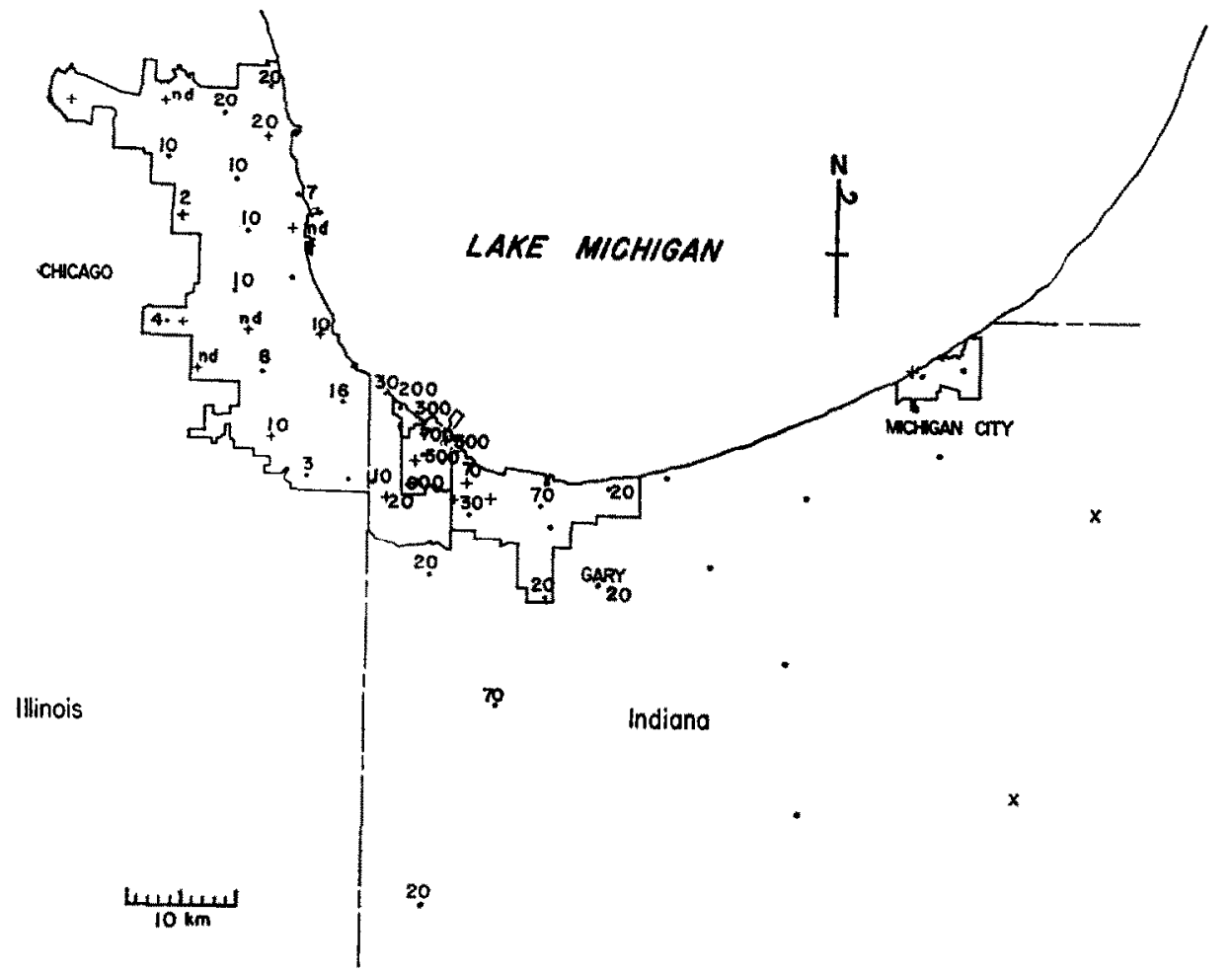

Fig. 5. Concentration of copper $\left(\mathrm{ng} \mathrm{m}^{-3} \times 0.1\right)$, Thursday, 6 June, 1968.

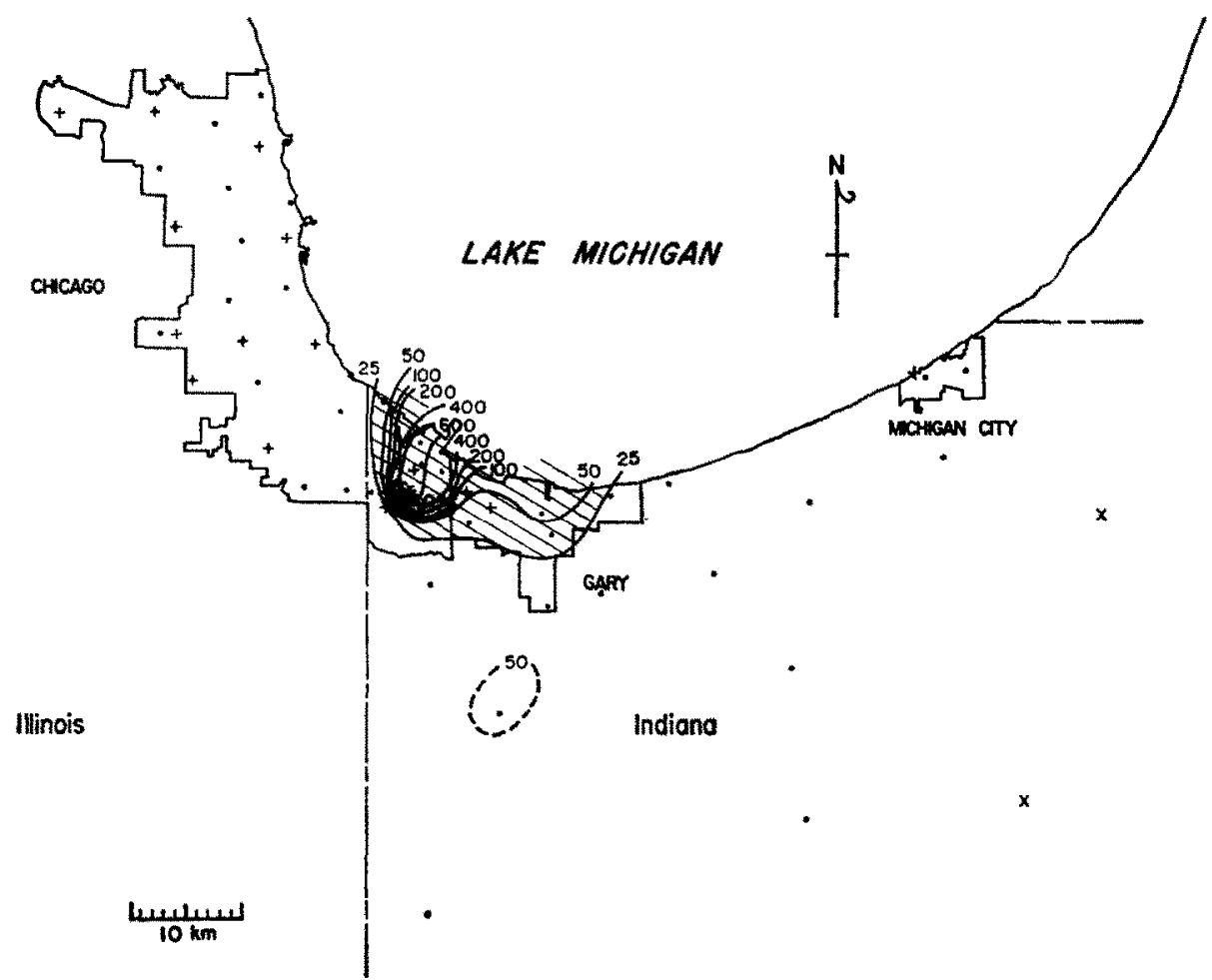

Fio. 6. Concentration of copper (ng $\mathrm{m}^{-3} \times 0.1$ ), Thursday, 6 June, 1968 . 


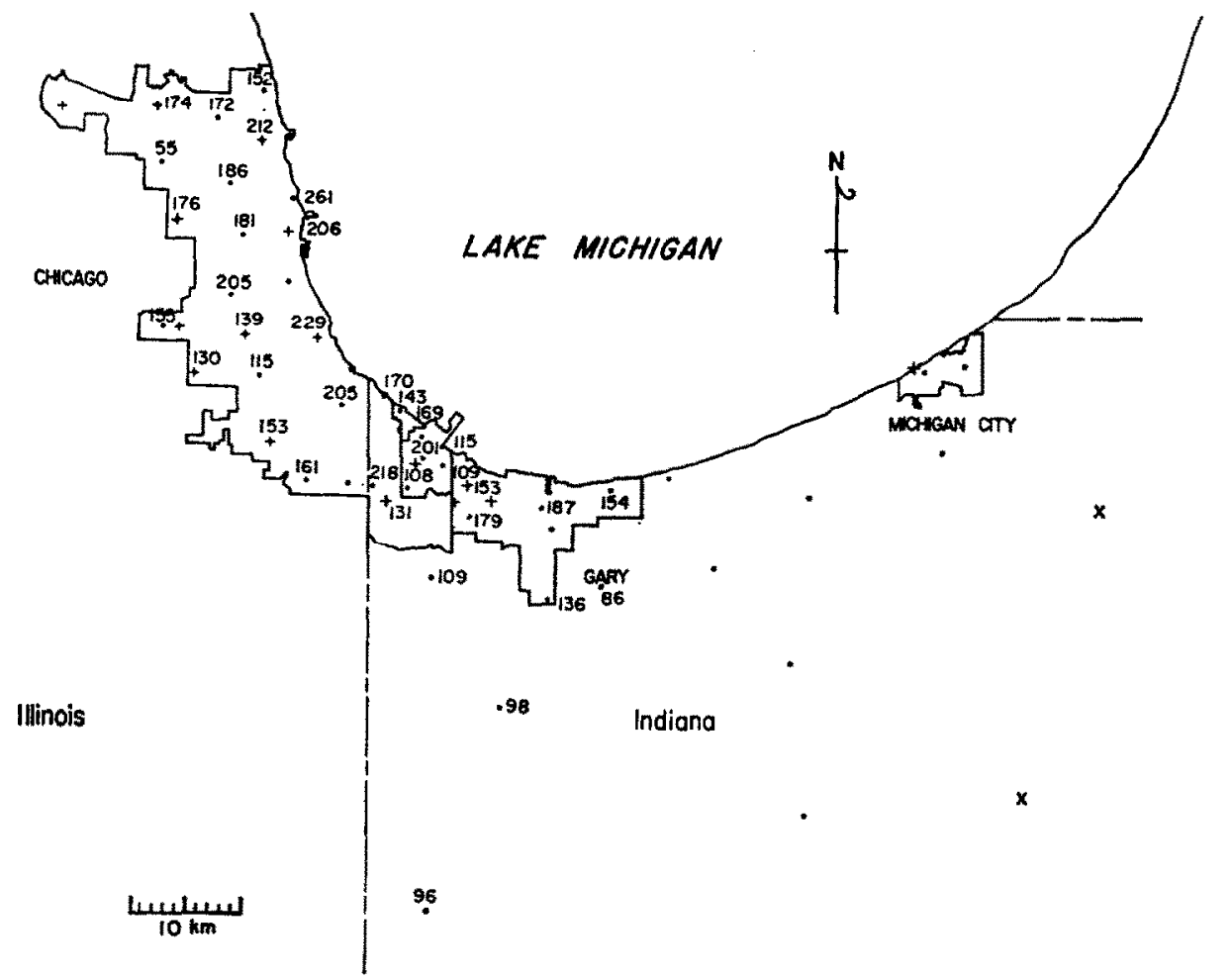

FiG. 7. Suspended particulate $\left(\mu \mathrm{g} \mathrm{m}^{-3}\right)$, Thursday, 6 June, 1968.

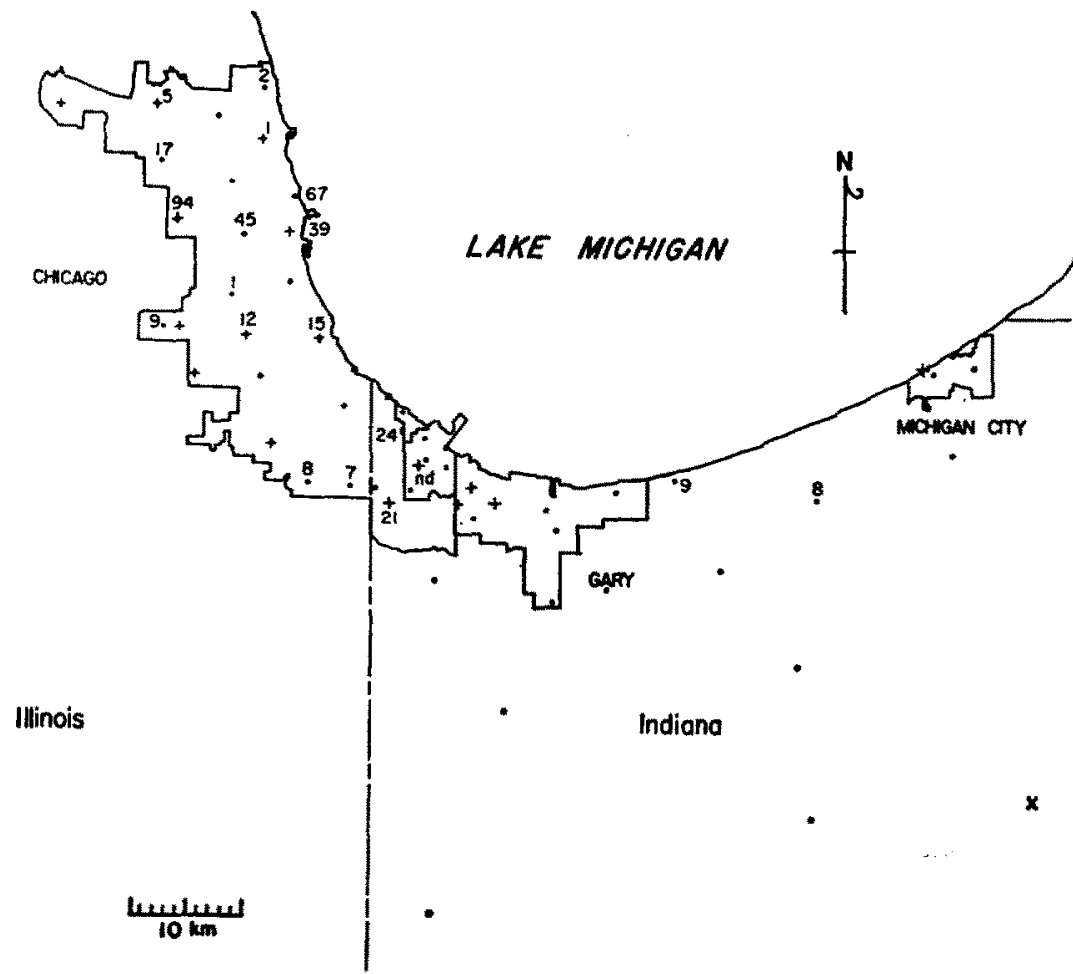

FiG. 8. Concentration of sulphur dioxide (ppb), Thursday, 6 June, 1968. 
Chicago, Indiana, where concentrations approach 100 times greater values. In Fig. 6 isopleths of the region show the systematic decrease in concentration with increasing distance from an apparently localized source region. Total suspended particulate, Fig. 7, does not reveal a maximum in this region but, like lead, seems to have a broad distribution with no obvious localized source. FIGURE 8 presents $\mathrm{SO}_{2}$ concentrations with a pattern apparently unrelated to the particulate data. All six days investigated show essentially the same location of a pronounced copper maximum and qualitatively similar behavior for the other variables illustrated in FIGS. 3-7. Bismuth data, obtained only for 21-22 May, show a low mean value near $1 \mathrm{ng} \mathrm{m}^{-3}$.

\section{DISCUSSION}

It is seen from the data that a large amount of scatter is present even in the broadly distributed suspended particulate and lead values. TABLE 7 summarizes the weighted

Table 7. Average meteorological characteristics

\begin{tabular}{rcrcccccccc}
\hline & $\begin{array}{c}\text { Wind } \\
\text { direction } \\
\text { (degrees) }\end{array}$ & $\begin{array}{c}\text { Range } \\
\text { (degrees) }\end{array}$ & $\begin{array}{c}\text { Speed } \\
\left(\mathrm{m} \mathrm{s}^{-1}\right)\end{array}$ & $\begin{array}{c}\text { Range } \\
\left(\mathrm{m} \mathrm{s}^{-1}\right)\end{array}$ & $\begin{array}{c}\text { Max. } \\
\left({ }^{\circ} \mathrm{C}\right)\end{array}$ & $\begin{array}{c}\text { Min. } \\
\left({ }^{\circ} \mathrm{C}\right)\end{array}$ & $\begin{array}{c}\text { Ave. } \\
\left({ }^{\circ} \mathrm{C}\right)\end{array}$ & $\begin{array}{c}\text { Precip. } \\
(\mathrm{mm})\end{array}$ & $\begin{array}{c}\text { Relative } \\
\text { humidity } \\
(\%)\end{array}$ \\
\hline 21 May & - & 360 & 3.3 & $\mathrm{C}-7$ & 13.9 & 8.3 & 11.1 & Trace & - \\
22 May & - & 280 & 3.5 & $\mathrm{C}-8$ & 16.7 & 6.1 & 11.7 & 2 & - \\
6 June & 180 & 40 & 5.3 & $2-8$ & 33.9 & 20.0 & 27.2 & None & 55 \\
20 June & 150 & 150 & 3.8 & $3-8$ & 25.6 & 13.3 & 19.4 & Trace & 61 \\
9 July & 210 & 170 & 6.8 & $4-10$ & 29.4 & 16.1 & 22.8 & Trace & 60 \\
8 Aug. & 200 & 260 & 3.9 & $2-16$ & 31.1 & 22.2 & 26.7 & 0.5 & 77 \\
29 Aug. & 090 & 150 & 4.2 & $3-6$ & 24.4 & 15.0 & 20.0 & & 58 \\
\hline
\end{tabular}

average wind direction, the total range of wind direction variability, mean speed and variability, temperature, rainfall, and relative humidity for the 6 sampling days. TABLE 8 gives the averages of the parameters broken into two groups of Chicago and Northwest Indiana, with Indiana copper values for stations 1-11 and 12-27 listed separately because of the large anomaly in East Chicago. Meteorological effects do not appear to be important in this study in affecting the observed patterns of concentration variation. However, except for $9 \mathrm{July}$, all days were influenced by cold air masses and variable wind directions. A lake breeze was observed on 29 August but the 24-h time gate does not permit an examination of this effect on trace metals. Thus, meteorology is still an unknown effect except for a bulk transport mechanism of pollutants.

The complexity of the study area is further seen in TABLE 9 giving the average values of ratios for each day separately for the Chicago and Indiana areas. The values of the ratios do not show large differences between the Indiana and the Illinois regions except for the large copper anomalies in Indiana. The mean $\mathrm{Cu} / \mathrm{Pb}$ ratio and mean $\% \mathrm{Cu} / \mathrm{S}$.P. are weighted strongly to $\mathrm{Cu}$ anomaly stations and show the large difference between Indiana and Chicago. $\mathrm{Cd} / \mathrm{Pb}, \% \mathrm{Cd} / \mathrm{S} . \mathrm{P}$., and $\% \mathrm{~Pb} / \mathrm{S} . \mathrm{P}$. do not show this difference as no large anomalies of $\mathrm{Cd}, \mathrm{Pb}$, or S.P. are found. The mean of the $\mathrm{Cd} / \mathrm{Cu}$ ratios is weighted strongly to the low $\mathrm{Cu}$ stations in Indiana and shows a similarity to the Chicago mean as expected. Thus, the mean $\mathrm{Cd} / \mathrm{Cu}$ ratio for all data is 0.11 and for the 
Table 8. Average concentrations for each day in Chicago and Northwest Indiana

\begin{tabular}{|c|c|c|c|c|c|c|}
\hline $\begin{array}{l}\text { Day, } \\
1968\end{array}$ & $\underset{\left(\mathrm{ng} \mathrm{m}^{-3}\right)}{\mathrm{Cd}}$ & $\begin{array}{c}\mathrm{Pb} \\
\left(\mathrm{ng} \mathrm{m}^{-3}\right)\end{array}$ & $\underset{\left(\mathrm{ng} \mathrm{m}^{-3}\right)}{\mathrm{Cu}^{*}}$ & $\underset{\left(\mu \mathrm{g} \mathrm{m}^{-3}\right)}{\mathrm{SP}}$ & $\begin{array}{c}\mathrm{SO}_{2} \\
(\mathrm{ppb})\end{array}$ & $\begin{array}{c}\mathrm{Bi} \\
\left(\mathrm{ng} \mathrm{m}^{-3}\right)\end{array}$ \\
\hline 21/22 May & & & 2000 & & & \\
\hline Ind. & 21 & 2000 & 180 & 92 & & 1.1 \\
\hline Ill. & 29 & 4300 & 300 & 130 & & 0.25 \\
\hline Both & 25 & 3300 & 750 & 110 & 19 & 0.63 \\
\hline 6 June & & & 3500 & & & \\
\hline Ind. & 8 & 830 & 390 & 140 & & \\
\hline Ill. & 9 & 1300 & 830 & 170 & & \\
\hline Both & 9 & 1100 & 970 & 160 & 20 & \\
\hline 20 June & & & 3900 & & & \\
\hline Ind. & 13 & 1700 & 260 & 170 & & \\
\hline IIl. & 10 & 2700 & 230 & 200 & & \\
\hline Both & 12 & 2200 & 1000 & 180 & 16 & \\
\hline 9 July & & & 1600 & & & \\
\hline Ind. & 19 & 810 & 130 & 84 & & \\
\hline Ill. & 9 & 810 & 99 & 83 & & \\
\hline Both & 14 & 810 & 510 & 84 & 12 & \\
\hline 8 Aug. & & & 4800 & & & \\
\hline Ind. & 5 & 1300 & 120 & 100 & & \\
\hline III. & 13 & 2600 & 250 & 180 & & \\
\hline Both & 9 & 1900 & 1300 & 146 & 9 & \\
\hline 29 Aug. & & & 4000 & & & \\
\hline Ind. & 12 & 1300 & 140 & 120 & & \\
\hline Ill. & 16 & 3100 & 220 & $180 \dagger$ & & \\
\hline Both & 14 & 2200 & 1200 & $156 \dagger$ & 17 & \\
\hline \multicolumn{7}{|c|}{ Totals: all data } \\
\hline Mean & & 1900 & 1000 & 150 & 18 & 0.63 \\
\hline Max. & 80 & 7000 & 10000 & 950 & 80 & 3.0 \\
\hline Min. & $\leqslant 5$ & 100 & $\leqslant 20$ & 30 & $\leqslant 0.5$ & $\leqslant .05$ \\
\hline
\end{tabular}

* Stations 1-10 and 11-22 listed separately.

$\dagger$ Station E excluded.

Chicago region is 0.12 for all 6 days. Correlation coefficients for all pairs and data points have been calculated (HARRISON, 1970) but owing to relatively large random errors for individual analyses are not as informative as the averages.

Elemental abundance patterns in an area-wide survey are useful in locating sources and may aid in making estimates of source strengths. We suggest from this study that $\mathrm{Pb}, \mathrm{Cd}$, and $\mathrm{Cu}$ over most of the Chicago and Northwest Indiana region come from area sources, but, in addition, $\mathrm{Cu}$ has a strong source or sources near stations 8 or 9 in Indiana. The source is persistent since the anomaly is seen on all 6 days of the survey, and tightness of the isopleths in FiG. 6 and other days implies a rather short atmospheric residence time and travel distance, seemingly unaffected by meteorological differences among the 6 days. Which urban or industrial activity is the actual source of this copper component may be determined by further investigation. We may ask whether conventional area sources of air pollution are adequate to account for $\mathrm{Pb}, \mathrm{Cd}$, and $\mathrm{Cu}$ outside 
Table 9. Average values of selected ratios for each day in Chicago and Northwest Indiana

\begin{tabular}{|c|c|c|c|c|c|c|c|}
\hline $\begin{array}{l}\text { Day } \\
1968\end{array}$ & $\mathrm{Cd} / \mathrm{Pb}$ & $\mathrm{Cd} / \mathrm{Cu}$ & $\mathrm{Cd} / \mathrm{SP}^{*}$ & $\mathrm{~Pb} / \mathrm{SP}^{*}$ & $\mathrm{Cu} / \mathrm{SP}^{*}$ & $\mathrm{Cu} / \mathrm{Pb}$ & $\mathrm{Cd} / \mathrm{SO}_{2}$ \\
\hline \multicolumn{8}{|l|}{ 21/22 May } \\
\hline Ind. & 0.012 & 0.099 & 0.023 & 2.2 & 1.7 & 0.89 & \\
\hline Ill. & 0.007 & 0.14 & 0.023 & 3.5 & 0.25 & 0.080 & \\
\hline Both & $(0.010)$ & $(0.12)$ & $(0.023)$ & (2.9) & $(0.92)$ & $(0.44)$ & $(9.0)$ \\
\hline \multicolumn{8}{|l|}{6 June } \\
\hline Ind. & 0.018 & 0.031 & 0.0098 & 0.57 & 1.49 & 3.4 & \\
\hline ill. & 0.011 & 0.16 & 0.0078 & 0.76 & 0.071 & 0.13 & \\
\hline Both & 0.014 & 0.10 & 0.0086 & 0.67 & 0.85 & 1.9 & 4.2 \\
\hline \multicolumn{8}{|l|}{20 June } \\
\hline Ind. & 0.021 & 0.11 & 0.022 & 1.1 & 0.87 & 1.3 & \\
\hline Ill. & 0.0044 & 0.087 & 0.0071 & 1.6 & 0.11 & 0.060 & \\
\hline Both & 0.012 & 0.10 & 0.014 & 1.4 & 0.54 & 0.78 & 3.8 \\
\hline \multicolumn{8}{|l|}{9 July } \\
\hline Ind. & 0.047 & 0.15 & 0.034 & 0.97 & 0.73 & 0.88 & \\
\hline Ill. & 0.018 & 0.15 & 0.019 & 1.0 & 0.13 & 0.15 & \\
\hline Both & 0.036 & 0.15 & 0.029 & 1.0 & 0.48 & 0.56 & 7.3 \\
\hline \multicolumn{8}{|l|}{8 August } \\
\hline Ind. & 0.014 & 0.053 & 0.013 & 1.1 & 2.4 & 2.6 & \\
\hline III. & 0.007 & 0.083 & 0.010 & 1.5 & 0.15 & 0.12 & \\
\hline Both & 0.009 & 0.070 & 0.011 & 1.3 & 1.3 & 1.5 & 3.8 \\
\hline \multicolumn{8}{|l|}{29 August } \\
\hline Ind. & 0.012 & 0.095 & 0.012 & 1.0 & 2.5 & 5.4 & \\
\hline Ill. & 0.007 & 0.096 & 0.013 & 1.7 & 0.11 & 0.082 & \\
\hline Both & 0.009 & 0.095 & 0.013 & 1.4 & 1.2 & 1.6 & 3.2 \\
\hline \multicolumn{8}{|l|}{ Totals: all data } \\
\hline Mean & 0.015 & 0.11 & 0.016 & 1.4 & 0.87 & 1.11 & 5.5 \\
\hline
\end{tabular}

* Per cent.

the anomalous region. WINCHESTER and NIFONG (1969) attempted to inventory some 30 individual trace elements of the Chicago-Northwest Indiana region from published information about fuel combustion and the steel and cement industries. Although only approximate these estimates may provide a basis for comparison of our results. TABLE 10 shows a summary of the data used to make these estimates, considering fuels to be the only important area for $\mathrm{Pb}, \mathrm{Cd}$, and $\mathrm{Cu}$ sources. These data lead us to expect ratios of elements coming from area fuel sources to be $\mathrm{Cd} / \mathrm{Cu} \sim 0.1, \mathrm{Cd} / \mathrm{Pb} \sim 0.006$, $\mathrm{Cu} / \mathrm{Pb} \sim 0.06$. Comparison of TABLE 9 shows approximate agreement of the observed means with these ratios, and it appears to be unnecessary at this time to invoke any other sources to account for these elements outside the $\mathrm{Cu}$ anomaly region. However, we consider it highly desirable to refine this comparison by use of improved data on the composition of particulate emission when they should become available.

Finally TABLE 11 is a comparison of our 6 day study with the National Air Sampling Network (NASN, 1966) stations in the area for 1963. Since our station 2 at or near the NASN station was available 5 of the 6 sampling days, we single this out especially for comparison. Cadmium falls within the variability presented by the NASN data, as 
TABle 10. Contributions of fuels to lead, CADMium, and Copper to Chicago area suspended PARTICULATE*

\begin{tabular}{lrccc}
\hline & Coal & Coke & Fuel oil & Gasoline \\
\hline Combustion estimate (Mtons $\mathrm{yr}^{-1}$ ) & 20 & 15 & 7 & 8 \\
Particulate emission estimate $\left(\mathrm{ktons}^{-1} \mathrm{yr}^{-1}\right.$ ) & 220 & 18 & 14 & $\dagger$ \\
Pb emission estimate (tons $\mathrm{yr}^{-1}$ ) & 300 & 22 & 30 & 1800 \\
Cd emission estimate (tons $\mathrm{yr}^{-1}$ ) & 11 & 1 & $\overline{26}$ & - \\
Cu emission estimate (tons $\mathrm{yr}^{-1}$ ) & 100 & 7 & - \\
\hline
\end{tabular}

* After Winchester and Nifong (1969). All units are metric.

$\dagger$ Assumed $2 \mathrm{~g} \mathrm{~Pb} \mathrm{gal}^{-1}$ and 25 per cent of emissions airborne.

TABle 11. Comparison With NASN 1963 data $\left(\mathrm{ng} \mathrm{m}^{-3}\right)$

\begin{tabular}{lcccc}
\hline & $\mathrm{Pb}$ & $\mathrm{Cd}$ & $\mathrm{Cu}$ & $\mathrm{Bi}$ \\
\hline NASN, Parke Co., rural & $24-57$ & $1.8-5.2$ & $17-80$ & $\leq 0.5$ \\
NASN, Beverly Shores, proximate & $100-200$ & $\mathrm{ND}-10$ & $30-50$ & $\leq 0.5$ \\
NASN, Hammond, urban & $100-1200$ & ND-55 & $30-180$ & $\leq 0.5$ \\
$\begin{array}{l}\text { This study, all stations* } \\
\text { This study, station 2, }\end{array}$ & $100-7000$ & $<5-80$ & $<20-10,000$ & $<0.05-3.0$ \\
$\quad$ Hammond* & $1000-3000$ & $<5-20$ & $100-1000$ & - \\
\hline
\end{tabular}

* Individual values reliable to a factor of 2 .

does bismuth. Lead and copper are both higher than the NASN data, a disagreement which we cannot explain adequately at this time. KNEIP et al. (1970) also find lead values in New York higher than NASN values and suggest that NASN determinations are erroneously low. We should point out that the NASN station is not located in the region of the anomalous copper and therefore leads to lower concentrations of $\mathrm{Cu}$ than we have found on the average in Northwest Indiana. In this study we have seen that area-wide studies can reveal (1) the general level of exposure of various pollutants, (2) previously unknown contaminants and sources, and (3) better locations for the placement of routine monitoring stations. Further studies of this type should be conducted under forecast meteorological conditions to gain knowledge of the mesoscale effects. The preliminary data suggest that the plumes diffuse rapidly from such areas and are lost in the proximate or non urban background levels.

Acknowledgements - Cooperation of air pollution control agencies in Chicago and Northwest Indiana in providing samples for analysis and the Thesis Parts Program at Argonne National Laboratory are gratefully acknowledged. This work was supported in part by the U.S. Public Health Service through grant AP-00585 and through an Air Pollution Traineeship award to one of us (P.R.H.).

\section{REFERENCES}

Brar S. S., Nelson D. M., Kanabrocki E. L., Moore C. E., Burnham C. D. and Hutton D. M. (1970). Thermal neutron activation analysis of particulate matter in surface air of the Chicago metropolitan area-one minute irradiations. Environ. Sci. Technol. 4, 50-54.

Harrison P. R. (1970) Ph.D. Thesis, University of Michigan.

Kneip T. J., Eisenbud M., Strehlow C. D. and Freudenthal P. C. (1970) Airborne particulates in New York City. J. Air Pollut. Control. Ass. 20 (3), 144-149. 
LEE J. and JERVIS R. E. (1968) Detection of pollutants in airborne particulates by activation analysis. Am. nucl. Soc. Trans. 11, 50-51.

MATSON W. R. (1968) Trace metals, equilibriumand kinetics of trace metal complexes in naturalmedia, Ph.D. Thesis, Department of Chemistry, Massachusetts Inst. of Tech.

National Air Sampling Networks (1966) Air Quality Data. U.S. Public Health Service.

TABOR E. C. and WARNer W. V. (1958) Archs Ind. Hith 17, 141-145. Am. Med. Ass.

WinCHESTER J. W. and NIFONG G. D. (1969) Water pollution in Lake Michigan by trace elements from pollution aerosol fallout. Paper WATR-34 presented at American Chemical Society, April.

Zeidberg L. D., Schueneman I. J. and Humphrey P. A. (1961) Air pollution and health: General description of a study in Nashville, Tennessee. J. Air Pollut. Control Ass. 11 (6), 289-297. 